\title{
BMJ Open Estimating the economic burden of cardiovascular events in patients receiving lipid-modifying therapy in the UK
}

Mark D Danese, ${ }^{1}$ Michelle Gleeson, ${ }^{1}$ Lucie Kutikova, ${ }^{2}$ Robert I Griffiths, ${ }^{1,3}$ Ali Azough, ${ }^{4}$ Kamlesh Khunti, ${ }^{5}$ Sreenivasa Rao Kondapally Seshasai, ${ }^{6}$ Kausik K Ray ${ }^{7}$

To cite: Danese MD, Gleeson M, Kutikova L, et al. Estimating the economic burden of cardiovascular events in patients receiving lipid-modifying therapy in the UK. BMJ Open 2016;6: e011805. doi:10.1136/ bmjopen-2016-011805

- Prepublication history and additional material is available. To view please visit the journal (http://dx.doi.org/ 10.1136/bmjopen-2016011805).

Received 9 March 2016 Revised 8 June 2016 Accepted 15 July 2016

CrossMark

For numbered affiliations see end of article.

Correspondence to

Dr Mark Danese;

mark@outins.com

\section{ABSTRACT}

Objectives: To characterise the costs to the UK National Health Service of cardiovascular (CV) events among individuals receiving lipid-modifying therapy.

Design: Retrospective cohort study using Clinical Practice Research Datalink records from 2006 to 2012 to identify individuals with their first and second CVrelated hospitalisations (first event and second event cohorts). Within-person differences were used to estimate CV-related outcomes.

Setting: Patients in the UK who had their first CV event between January 2006 and March 2012.

Participants: Patients $\geq 18$ years who had a CV event and received at least 2 lipid-modifying therapy prescriptions within 180 days beforehand.

\section{Primary and secondary outcome measures:}

Direct medical costs $(2014 £)$ were estimated in 3 periods: baseline (pre-event), acute (6 months afterwards) and long-term (subsequent 30 months). Primary outcomes included incremental costs, resource usage and total costs per period.

Results: There were 24093 patients in the first event cohort of whom 5274 were included in the second event cohort. The mean incremental acute CV event costs for the first event and second event cohorts were: coronary artery bypass graft/percutaneous transluminal coronary angioplasty (CABG/PTCA) £5635 and £5823, myocardial infarction £4275 and £4301, ischaemic stroke £3512 and $£ 4572$, heart failure £2444 and £3461, unstable angina $£ 2179$ and £2489 and transient ischaemic attack £1537 and £1814. The mean incremental long-term costs were: heart failure £848 and £2829, myocardial infarction £922 and $£ 1385$, ischaemic stroke $£ 973$ and $£ 682$, transient ischaemic attack $£ 705$ and $£ 1692$, unstable angina $£ 328$ and £677, and CABG/PTCA £-368 and £599.

Hospitalisation accounted for $95 \%$ of acute and $61 \%$ of long-term incremental costs. Higher comorbidity was associated with higher long-term costs.

Conclusions: Revascularisation and myocardial infarction were associated with the highest incremental costs following a CV event. On the basis of real-world data, the economic burden of CV events in the UK is substantial, particularly among those with greater comorbidity burden.

\section{Strengths and limitations of this study}

- All estimates were calculated using real-world data for the UK.

- Incremental costs were estimated using withinperson differences to minimise confounding.

- All patients received lipid-modifying therapy, maximising the relevance for this population.

- Censoring in the data may affect the cost estimates, particularly for long-term costs.

- The estimates were based on external cost data applied to general practitioner and inpatient hospitalisation usage, with limited information on outpatient specialist care.

\section{INTRODUCTION}

Cardiovascular (CV) disease is a major cause of premature death worldwide and an important source of disability. Elevated levels of blood lipids represent a major risk factor for the development of coronary heart disease including myocardial infarction (MI) and angina, as well as other CV events including stroke, transient ischaemic attack (TIA) and peripheral arterial disease. A history of these events places individuals at higher risk of experiencing subsequent $\mathrm{CV}$ events. $^{1}$

A meta-analysis of 21 trials of $>170000$ randomised patients demonstrated that the incidence of major $\mathrm{CV}$ events was reduced by $\sim 25 \%$ for each $\mathrm{mmol} / \mathrm{L}$ reduction in lowdensity lipoprotein (LDL) cholesterol. ${ }^{2}$ Therefore, reducing serum cholesterol levels is the cornerstone of $\mathrm{CV}$ disease prevention efforts. ${ }^{3}$ Drug treatment with lipid-modifying therapy to lower lipids, especially statins, is commonly used with the goal of reducing population $\mathrm{CV}$ event rates. In addition to the potential public health benefits, lower $\mathrm{CV}$ event rates from reducing LDL should also have economic benefits in terms of cost 
offsets from prevented CV events. However, many patients do not attain target lipid levels for a variety of reasons including the use of lower than optimal doses, non-compliance and very high levels of LDL that cannot be sufficiently lowered with existing therapies. Thus, these individuals carry a higher risk of $\mathrm{CV}$ events such as MI and stroke that could be reduced with greater LDL reductions and/or improved adherence. ${ }^{13}$

In the UK, studies of the cost of CV events have not been conducted until now, which is most likely due to two challenges. First, cost information is generally not included with clinical data in the UK. Therefore, analysing costs requires a complicated process of merging information from disparate sources. Second, the linkage to Hospital Episode Statistics (HES) data has relatively recently become available to provide the detailed information necessary for estimating costs based on Healthcare Resource Groups (HRGs).

Until now, $\mathrm{CV}$ event cost estimates have been based primarily on expert opinion regarding usage, combined with UK-specific costs for the resources. ${ }^{4}$ Although studies of CV event costs have been previously published, they are generally limited to analysing short-term costs using US data sources. ${ }^{5-10}$ While these studies include patients receiving lipid-modifying therapy, they tend to focus on patients hospitalised for CV events, or patients with atherosclerosis, hypertension or acute coronary syndrome; they do not provide estimates specific to the lipidmodifying therapy population which may experience a different distribution of CV events with different patterns of resource use. Having analyses in the lipid-modifying therapy population provides useful evidence to physicians, payers or other decision-makers interested in the burden of CVD and related health economic analyses.

The present study was designed to provide evidencebased estimates of healthcare resource use (HRU) and costs specifically for the lipid-modifying therapy population. The primary objective was to estimate the shortterm and long-term HRU and costs associated with specific new CV events (including initial and subsequent events) in UK patients receiving lipid-modifying therapy. A secondary objective was to explore the effects of comorbidity burden on these costs.

\section{METHODS}

\section{Study overview}

This was a retrospective cohort study to estimate resource usage and direct medical costs after surviving a first or second CV event in patients receiving lipidmodifying therapy prior to the event. Primary care data were obtained from electronic medical records in the Clinical Practice Research Datalink (CPRD), and inpatient data were obtained from the linked HES data.

\section{Data sources}

This was an observational cohort study. ${ }^{11}$ The CPRD contains anonymised information from general practitioners (GP) on demographics, symptoms, diagnoses, test results and referrals to secondary care, and is a widely used database globally. ${ }^{12}$ These patients are broadly representative of the UK general population in terms of age, sex and ethnicity. At the time this study was conducted, the CPRD-HES linkage included hospitalised care but did not include data for outpatients or for accident and emergency visits.

\section{Patient populations}

The study population included adult patients ( $\geq 18$ years) who were alive and observable in the CPRD and HES data as of 1 January 2005, and were hospitalised for their first CV event between January 2006 and 31 March 2012 (end of HES observation period). Only patients who had a CV event in the HES data were included in the study. CV events were defined as hospitalisations with a primary International Classification of Diseases, 10th Revision (ICD-10) diagnosis code for MI, ischaemic stroke (IS), heart failure (HF), TIA or unstable angina (UA), or other hospitalisations for revascularisation that included coronary artery bypass graft (CABG) or percutaneous transluminal coronary angioplasty (PTCA). Percutaneous coronary intervention (angioplasty with stenting) was included with PTCA. Patients with a history of an acute event (MI or IS) in the CPRD data before 2006 were also excluded. Prescription records for lipid-modifying therapy were used to identify patients receiving treatment to reduce serum lipid levels. To ensure that patients were receiving lipid-modifying therapy prior to their first CV event, they had to have received at least two prescriptions for lipidmodifying therapy in the 180 days prior to the index date. Lipid-modifying therapy included statins, ezetimibe, fibrates, nicotinic acid and bile acid sequestrants.

The date of the event that qualified the patient to be in a cohort was defined as the 'index' date. To rule out previous events more accurately, patients were required to have at least 12 months of data prior to the CV event date; we also required at least 30 days of follow-up afterwards to properly categorise the index event. Patients who met these inclusion criteria were included in the first event cohort. In addition, a second event cohort was created using patients who had a subsequent CV event, the date of which was their index date (see online supplementary materials figure $\mathrm{S} 1$ ).

The medical history of any of the following conditions in the CPRD was also captured: diabetes, peripheral artery (vascular) disease, abdominal aortic aneurysm, TIA, ischaemic heart disease (excluding MI or UA), carotid artery disease or angina pectoris. These conditions were identified using READ codes based on code lists from the Quality Outcomes Framework (QOF) where possible. ${ }^{13}$

\section{Study design}

This was a pre-post design, using patients as their own controls, and was used to reduce the influence of 
potential confounders that could have influenced the $\mathrm{CV}$ event costs. Patients were selected at the time they had their qualifying CV event. To estimate costs related to first and second $\mathrm{CV}$ events compared with no $\mathrm{CV}$ event, we estimated costs after each CV event and calculated the incremental difference from the period before the first $\mathrm{CV}$ event.

The assessment of demographics and comorbidity information was based on information present at the time of the index CV event. Baseline usage and costs for all cohorts were estimated during the 12-month period prior to the first CV event for all patients. The follow-up period for the assessment of HRU and costs started with the date of the index $\mathrm{CV}$ event and continued for 36 months, or until the end of data availability within the HES database (31 March 2012). Patients were also censored at date of death, date of a subsequent CV hospitalisation event, date of last known up-to-standard CPRD record for the patient in the practice or 31 March 2012, whichever came first.

For the subset of first event cohort patients who had a second CV event, the baseline period was the 12-month period before the second event and the follow-up period was the 36-month period after it. Since follow-up was censored at subsequent $\mathrm{CV}$ events, costs were not double-counted between the first event and second event cohorts. This facilitated reporting costs for first and second events separately as well as reporting pooled results.

The primary time periods for the analyses were the first 6 months after the index event ('acute period'), and the subsequent 30 months after the acute period ('long-term period'). These intervals were selected to align with time frames provided in a National Institute for Health and Care Excellence (NICE) clinical guideline for lipid modification. ${ }^{4}$ Long-term outcomes were annualised for easier interpretation. For analyses of incremental usage and cost in this cohort, the 12-month period before the first CV event was used as the baseline for calculating all cost differences, including those in the second event cohort. This was done to have a consistent reference cost for the calculation of incremental costs across cohorts.

\section{Study end points}

\section{Primary end points}

The primary end points in this study were resource usage and direct medical costs for the acute period after a $\mathrm{CV}$ hospitalisation, and the subsequent long-term period. Both total and incremental CV-related costs were estimated, including hospitalisations (HES), outpatient referrals (CPRD), primary care office visits (CPRD) and medications (CPRD). Medications included lipid-lowering therapy, antihypertensive therapy, antithrombotic therapy and antidiabetic therapy (see online supplementary materials table $\mathrm{S} 1$ ).

All events within 30 days after the initial event were used to categorise the event type. Multiple events occurring within this 30-day window were assigned to a group using the highest event from the following hierarchy: MI, UA, IS, CABG, PTCA, HF and TIA. (For example, someone with a TIA followed by MI, then CABG within a 30-day window would be assigned to the MI group.) This ensured that temporally close observations were not double-counted, and reduced censoring due to subsequent events. All hospitalisations were identified using ICD-10 codes for diagnoses and Office of Population, Censuses and Surveys (OPCS) codes for procedures (see online supplementary materials table S2).

\section{Costing}

\section{Data sources}

Unit costs were attributed to the identified resource use category for each individual patient and aggregated across all patients. Unit costs for secondary care (hospitalisation) and drugs were derived from the 2014 UK National Health Service (NHS) sources to ensure transparency and relevancy in the source of cost data. ${ }^{14}{ }^{15}$ Primary care unit costs were estimated from the Personal Social Services Research Unit 2014 costs. ${ }^{16}$

Office visits in surgery or clinic were priced at $£ 46$ (11.7 min visit) and telephone consultations at £28 (7.1 min consult) based on 2014 data. ${ }^{15}$ Outpatient referrals listed in the CPRD data were assumed to have occurred and costs were assigned using NHS reference costs for 2013-2014 according to specialty type. ${ }^{4}$ Costs for hospitalisations were based on HRG Reference Costs and were assigned using the 2013/2014 HRG4+ Reference Costs Grouper software. ${ }^{14}$ All available diagnosis and procedure codes were used to assign the hospital cost. Drug costs were based on the NHS Electronic Drug Tariff for England and Wales using data from September 2014 where possible, augmented with other months in 2014 for drugs that did not have a September 2014 cost. Generic prices were used where possible. ${ }^{17}$

\section{Analyses}

Estimation of patient costs was performed by multiplying the quantity of each resource used by the corresponding unit cost of the resource. Costs were grouped into hospital, drug and office visit (including outpatient referral). Acute and long-term incremental and total costs were also stratified by the type of index event. Exploratory analyses of acute and long-term incremental costs were stratified by the Charlson Comorbidity Index ${ }^{18}$ and by age group. Comorbid conditions for the Charlson Comorbidity Index were identified using a study that provided UK-specific code lists for each condition. $^{19}$

Since patients were censored during the follow-up period, we used inverse probability weighting to account for censoring and death. ${ }^{20} 21$ This facilitated the summation of costs, and the calculation of incremental costs over the time intervals in the study, even when different numbers of patients were present in each interval. 
To combine costs and align with the acute and longterm periods, the first year costs were divided into two 6-month time intervals, while the remaining costs were divided into annual time intervals. Death was treated as a censoring event to align with economic model inputs that require the annual cost of care for survivors (ie, attenuation of costs due to death is incorporated into the economic models ${ }^{22}$ ). Variances were estimated using bootstrapping of the inverse probability weighted estimates for each time interval. Usage estimates were analysed similarly to cost estimates to account for censoring and death.

Patient demographic and clinical characteristics were described using means and SDs for continuous variables, and percentages for categorical variables. Analyses of mortality rates were based on deaths from all causes divided by person-years of follow-up until death or a censoring event, and are expressed per 100 person-years. All analyses were conducted in $\mathrm{R}$ (V.3.1.3). ${ }^{23}$

\section{RESULTS}

\section{Cohort overview}

Baseline characteristics and mortality rates are summarised by cohort in table 1 . There were 24093 patients in the first event cohort. Of these, 5274 (22\%) had a subsequent $\mathrm{CV}$ event in our study time horizon and were also included in the second event cohort. The mean age at the time of their $\mathrm{CV}$ event was 73 years in both cohorts, the proportion of males was $59 \%$ and $60 \%$ respectively, the mean body mass index was $29 \mathrm{~kg} / \mathrm{m}^{2}$ in both cohorts, and baseline LDL cholesterol levels were 2.3 and $2.2 \mathrm{mmol} / \mathrm{L}$, respectively. Within 6 months of the index event, $11.5 \%$ of the first event and $13.9 \%$ of the second event cohort died. All-cause mortality rates were higher in the 6-month acute period (28.5 and 36.5 per 100 person-years, respectively), and lower in the 30-month long-term period (5.4 and 6.2 per 100 personyears, respectively). Figure 1 shows the distribution of $\mathrm{CV}$ event type for each of the cohorts. Approximately $97 \%$ of patients were included in the study based on receiving a statin as their lipid-modifying therapy prescription within 180 days prior to their first CV event (table 1).

\section{CV event costs}

The incremental CV event costs in the acute period were $£ 3504$ in the first event cohort and $£ 3968$ in the second event cohort (table 2). The incremental cost for both cohorts combined was $£ 3577$. Hospitalisation costs were $£ 3338$ and £3737, respectively, and £3400 averaged across both cohorts. The incremental costs in the subsequent 30 months were $£ 361$ and £1018 per year, respectively, and £439 averaged across both cohorts. Hospitalisation accounted for $95 \%$ of acute and $61 \%$ of long-term incremental costs.

In terms of index event subgroups, the highest acute period incremental CV event costs were for the CABG/
PTCA, MI and patients with IS in both cohorts (table 2). The mean costs for the first event and second event cohorts were as follows: CABG/PTCA $£ 5635$ and $£ 5823$, MI $£ 4275$ and £4301, IS £3512 and £4572, HF £2444 and $£ 3461$, UA £2179 and £2489 and TIA £1537 and £1814. The subsequent long-term incremental costs (per year) were more heterogeneous and were as follows for the first event and second event cohorts, respectively: HF $£ 8848$ and £2829, MI £922 and £1385, IS £973 and £682, TIA $£ 705$ and £1692, UA £328 and £677, and CABG/ PTCA £-368 and £599.

Figure 2 shows stratified incremental costs for each cohort by comorbidity status, and table 3 shows the corresponding rate of office visits, subsequent $\mathrm{CV}$ event rate, death rate and the initial CV hospitalisation length of stay. In the first event cohort, higher comorbidity scores from the Charlson Comorbidity Index were associated with comparable acute period costs and higher long-term period costs. In the second event cohort, acute and long-term annual costs increased with the Charlson score. These trends were generally mirrored by corresponding usage and event rate patterns in table 3. Costs stratified by age group did not vary as systematically (see online supplementary materials figure S2).

Overall and hospital-specific total (ie, nonincremental) costs in the 12-month baseline period, the 6-month acute period and the 30-month long-term period are provided in table 2. In terms of total costs within the baseline period, the highest was for patients with HF, whose annual cost was $£ 3149$ in the first event cohort and $£ 2738$ in the second event cohort. In the acute period, the highest total cost was for CABG/ PTCA, which was $£ 6843$ in the first event cohort and $£ 6630$ in the second event cohort. In the long-term period, the highest annualised cost was in patients with HF, which was $£ 3608$ in the first event cohort and $£ 5554$ in the second event cohort.

In the acute period after the index event, there were 5.0 and 6.1 more office visits as well as 1.5 and 1.6 more non-CV hospitalisations per person and $1 \mathrm{CV}$ hospitalisation per person for the first event and second event cohorts, respectively. During the long-term period afterwards, there were 2.9 and 4.8 more office visits as well as 0.2 and 0.5 more non-CV hospitalisations compared with the baseline period. The mean length of stay for the index hospitalisation was shortest for UA (4.5 and 4.9 days) and longest for IS (22.5 and 26.7 days).

\section{DISCUSSION}

Estimating resource use and the associated costs of managing CVD is important for estimating the value of interventions that reduce the risk of CV events. Until now, these estimates have been based on expert opinion regarding usage, combined with UK-specific costs for the resources. ${ }^{12}$ To the best of our knowledge, this is the first study both to measure usage in lipid-modifying therapy patients with $\mathrm{CV}$ events using comprehensive 
real-world data and to combine this information with costs from the UK perspective.

These data show that the cost of care for patients experiencing a CV event was generally very high in the acute period following the event. In particular, patients with a second GV event had higher incremental CV-related costs. These incremental CV-related costs were generally much smaller in the long-term period, although they were almost always still positive (ie, greater than the baseline pre-CV event period). The

\begin{tabular}{|c|c|c|c|c|c|c|c|}
\hline \multirow{3}{*}{$\begin{array}{l}\text { Variable } \\
\text { Continuous variables }\end{array}$} & & \multicolumn{6}{|l|}{ Cohort } \\
\hline & & \multicolumn{3}{|c|}{ First event ( $n=24093)$} & \multicolumn{3}{|c|}{ Second event $(n=5274)$} \\
\hline & & $\overline{\mathbf{N}}$ & Mean & SD & $\overline{\mathbf{N}}$ & Mean & SD \\
\hline Age at index & & 24093 & 72.7 & 11.1 & 5274 & 72.8 & 10.8 \\
\hline BMI & & 15005 & 28.8 & 5.7 & 3457 & 28.7 & 5.6 \\
\hline SBP & & 23107 & 136.1 & 19.2 & 5119 & 132.6 & 20.1 \\
\hline LDL cholesterol & & 13247 & 2.4 & 2.3 & 2803 & 2.2 & 0.9 \\
\hline Total cholesterol & & 19693 & 4.4 & 1.4 & 4267 & 4.2 & 1.4 \\
\hline Triglycerides & & 15064 & 1.6 & 1.2 & 3143 & 1.6 & 1.0 \\
\hline Categorical variables & Level & $\mathbf{N}$ & Per cent & & $\mathbf{N}$ & Per cent & \\
\hline \multirow{5}{*}{ Lipid-modifying therapy (before or on index date) ${ }^{*}$} & Low-dose statin & 1918 & 8.0 & & 428 & 8.1 & \\
\hline & Moderate-dose statin & 18556 & 77.0 & & 3980 & 75.5 & \\
\hline & High-dose statin & 2886 & 12.0 & & 695 & 13.2 & \\
\hline & Ezetimibe & 465 & 1.9 & & 104 & 2.0 & \\
\hline & All others & 268 & 1.1 & & 67 & 1.3 & \\
\hline \multirow[t]{7}{*}{ Year of index event } & 2006 & 3948 & 16.4 & & 425 & 8.1 & \\
\hline & 2007 & 3975 & 16.5 & & 782 & 14.8 & \\
\hline & 2008 & 3858 & 16.0 & & 877 & 16.6 & \\
\hline & 2009 & 4023 & 16.7 & & 986 & 18.7 & \\
\hline & 2010 & 3801 & 15.8 & & 980 & 18.6 & \\
\hline & 2011 & 3630 & 15.1 & & 987 & 18.7 & \\
\hline & 2012 & 858 & 3.6 & & 237 & 4.5 & \\
\hline \multirow[t]{4}{*}{ Age group } & $<60$ & 3170 & 13.2 & & 652 & 12.4 & \\
\hline & $60-69$ & 4892 & 20.3 & & 1007 & 19.1 & \\
\hline & $70-79$ & 8864 & 36.8 & & 2092 & 39.7 & \\
\hline & $\geq 80$ & 7167 & 29.8 & & 1523 & 28.9 & \\
\hline \multirow[t]{2}{*}{ Gender } & Male & 14221 & 59.0 & & 3177 & 60.3 & \\
\hline & Female & 9872 & 41.0 & & 2097 & 39.8 & \\
\hline \multirow[t]{3}{*}{ Smoking status } & Current & 2867 & 11.9 & & 488 & 9.3 & \\
\hline & Former & 9020 & 37.4 & & 2248 & 42.6 & \\
\hline & Never & 6483 & 26.9 & & 1375 & 26.1 & \\
\hline \multirow[t]{3}{*}{ Charlson Comorbidity Index (score) } & None & 7199 & 29.9 & & 831 & 15.8 & \\
\hline & One & 5378 & 22.3 & & 1029 & 19.5 & \\
\hline & Two or higher & 11516 & 47.8 & & 3414 & 64.7 & \\
\hline \multirow{5}{*}{ Risk factors } & Hypertension & 10291 & 42.7 & & 2364 & 44.8 & \\
\hline & Diabetes & 7343 & 30.5 & & 1905 & 36.1 & \\
\hline & COPD & 1958 & 8.1 & & 524 & 9.9 & \\
\hline & CKD & 6038 & 25.1 & & 1815 & 34.4 & \\
\hline & $\mathrm{AF}$ & 3085 & 12.8 & & 1014 & 19.2 & \\
\hline \multirow[t]{6}{*}{ CV conditions } & AAA & 143 & 0.6 & & 25 & 0.5 & \\
\hline & Angina & 7239 & 30.1 & & 1861 & 35.3 & \\
\hline & PVD & 1972 & 8.2 & & 524 & 9.9 & \\
\hline & TIA & 2005 & 8.3 & & 442 & 8.4 & \\
\hline & Cardiac ischaemia & 10261 & 42.6 & & 2508 & 47.6 & \\
\hline & Carotid stenosis & 223 & 0.9 & & 54 & 1.02 & \\
\hline \multirow[t]{3}{*}{ Other medications } & Antihypertensive & 20973 & 87.1 & & 4979 & 94.4 & \\
\hline & Antithrombotic & 18818 & 78.1 & & 4901 & 92.9 & \\
\hline & Antidiabetic & 6073 & 25.2 & & 1599 & 30.3 & \\
\hline Mortality rate (per 100 person-years) & Time interval & Person years & Died & Rate & Person years & Died & Rate \\
\hline Acute & $0-6$ months & 9686 & 2764 & 28.5 & 2006 & 733 & 36.5 \\
\hline Long term & $7-36$ months & 22798 & 1240 & 5.4 & 3967 & 246 & 6.2 \\
\hline
\end{tabular}

*Lipid-modifying therapy refers to the closest prescription to the index date within 180 days prior to it. See online supplementary materials for definitions of medication categories.

AAA, abdominal aortic aneurysm; AF, arterial fibrillation; BMI, body mass index; CKD, chronic kidney disease; COPD, chronic obstructive pulmonary disease; CV, cardiovascular; LDL, low-density lipoprotein; PVD, peripheral vascular disease; SBP, systolic blood pressure; TIA, transient ischaemic attack. 
Figure 1 Distribution of types of index events by cohort. CABG, coronary artery bypass graft; $\mathrm{HF}$, heart failure; IS, ischaemic stroke; $\mathrm{MI}$, myocardial infarction; PTCA, percutaneous transluminal coronary angioplasty; TIA, transient ischaemic attack; UA, unstable angina.

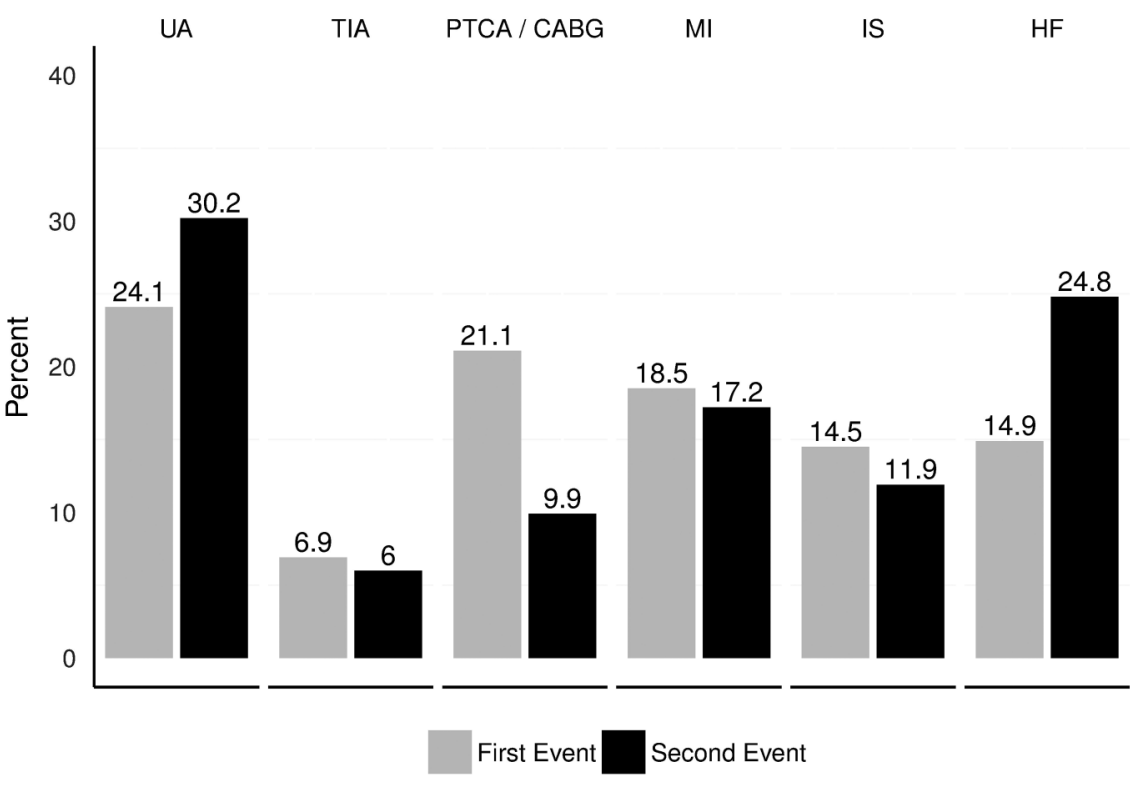

exception to this trend was for CABG/PTCA in the first event cohort, which suggests that this procedure might have long-term cost offsets in patients who had these procedures in the absence of one of the other qualifying CV events. The incremental long-term cost in the second event cohort was the lowest in patients with CABG/PTCA.

It is also interesting that the first event and second event cohorts often had similar incremental acute CV-related costs. This is most likely related to hospitalisation costs, which were the dominant cost driver during our study's time horizon. In addition, it appears that comorbidity burden is an important contributor to incremental long-term costs as shown in figure 2. The modest association of age and long-term costs in online supplementary materials figure S2 is most likely caused by increases in comorbidity burden with increasing age in the three younger age groups. We were not able to evaluate the effects of compliance and persistence with lipid-modifying therapy on clinical and economic outcomes. However, a recent study showed that $43 \%$ of patients discontinued their statins within 12 months of initiation, highlighting the importance of doing such research in the future. ${ }^{24}$

It is important to highlight the clinical implications of these results. CV events are multifactorial, and CV event costs might vary according to the CV risk factors of the patient population. Since our focus was on estimating costs in patients at risk of atherosclerotic vascular events, we required patients to have received at least two lipidmodifying therapy prescriptions within 180 days. While one would expect CV event costs to be similar in other populations, these results are most relevant for patients receiving lipid-modifying therapy. The fact that many of these patients, most of whom were receiving antithrombotic and antihypertensive treatment, went on to experience subsequent CV events highlights the limitations of available interventions in a population with substantial need. Furthermore, the progressively more costly results by higher comorbidity risk score indicate that comorbid conditions are another important factor for these patients.

The primary value of these analyses is that they are CPRD data reflect the medical records of $\sim 6 \%$ of all patients in clinical practice in the UK, and are considered to be a very reliable source of longitudinal patient data, supporting over 1500 publications. ${ }^{25}{ }^{26}$ Patients were included based on hospitalisations in the HES data, so they align with intense resource usage that is relevant to supporting economic modelling efforts. This is in contrast to events recorded in the CPRD GP data that may not reflect hospitalisation, depending on the reason for recording a diagnosis code at a particular office visit. ${ }^{27}$ Finally, the perspective of the NHS was used for costing where possible, to ensure alignment with the payer for the majority of services provided in the UK.

It must be emphasised, though, that our findings merit careful interpretation in the light of potential limitations of our data and the analyses. For instance, we used external data sources for 2014 for costing and these may not reflect the actual resources used for the patients in this study. This is most likely to affect the hospitalisation costs, since the costs of office visits and drugs are much smaller and less variable. We included the cost of referral visits, based on referrals recorded in the CPRD referral file. We cannot ascertain whether these visits actually occurred, so the usage of these services (and hence the costs) may be overestimated. On the contrary, not every visit to a specialist is recorded by the GP, causing cost underestimation and counterbalancing the potential bias to some degree.

Importantly, we included all hospitalisations for each patient in our usage and cost analyses, and not just CV hospitalisations. This may lead to more noise in the data if there were to be no relationship between the CV events derived from current real-world data in the UK. The 
Table 2 CV event-related costs by cohort and index event

\begin{tabular}{|c|c|c|c|c|c|c|c|}
\hline \multirow[b]{2}{*}{ Cohort } & \multirow[b]{2}{*}{ Group } & \multirow[b]{2}{*}{$\mathbf{n}$} & \multicolumn{3}{|l|}{ Total costs (in $£$ ) } & \multicolumn{2}{|c|}{ Incremental total costs (in £) } \\
\hline & & & $\begin{array}{l}\text { Baseline (1 year } \\
\text { before first CV event) } \\
\text { (mean (SE)) }\end{array}$ & $\begin{array}{l}\text { Months 1-6 } \\
\text { (mean (SE)) }\end{array}$ & $\begin{array}{l}\text { Months 7-36 } \\
\text { annualised } \\
\text { (mean (SE)) }\end{array}$ & $\begin{array}{l}\text { Months 1-6 } \\
\text { (mean (SE)) }\end{array}$ & $\begin{array}{l}\text { Months } 7-36 \\
\text { annualised (mean (SE)) }\end{array}$ \\
\hline \multirow[t]{8}{*}{ First event } & Myocardial infarction & 4468 & $2266.34(163.45)$ & $5297.59(115.51)$ & $2350.66(165.54)$ & $4275.41(102.41)$ & $922.43(155.58)$ \\
\hline & Unstable angina & 5801 & $1965.61(86.76)$ & $3133.12(61.44)$ & $2081.84(65.15)$ & $2179.24(48.51)$ & $328.45(61)$ \\
\hline & Ischaemic stroke & 3489 & $1962.42(119.73)$ & $4389.27(102.29)$ & $2526.63(276.42)$ & $3512.25(102.82)$ & $972.62(257.48)$ \\
\hline & PTCA/CABG* & 5082 & 2412.2 (95.69) & $6843.38(94.67)$ & $1855.11(110.02)$ & 5635.19 (87.29) & $-368.26(103.58)$ \\
\hline & Heart failure & 3596 & $3148.72(136.77)$ & $3988.86(111.87)$ & $3607.86(313.16)$ & $2443.58(95.22)$ & $847.55(247.64)$ \\
\hline & Transient ischaemic attack & 1657 & $2109.76(215.46)$ & $2488.83(89.42)$ & $2362.10(152.91)$ & $1536.88(69.22)$ & $704.76(147.47)$ \\
\hline & All patients (hospital) & 24093 & $1565.41(56.72)$ & 4059.91 (38.39) & $1432.92(59.15)$ & $3337.51(37.75)$ & $203.18(48.2)$ \\
\hline & All patients (total) & 24093 & $2301.34(57.26)$ & $4594.16(39.01)$ & $2262.92(60.37)$ & 3504.01 (38.04) & 361.11 (48.79) \\
\hline \multirow[t]{8}{*}{ Second event } & Myocardial infarction & 769 & 2611.88 (326.29) & 5785.47 (353.52) & 3887.74 (909.42) & 4301.01 (330.02) & $1384.51(622.14)$ \\
\hline & Unstable angina & 1347 & 2795.44 (398.16) & 3925.66 (204.31) & 2844.49 (247.33) & 2489.48 (132.63) & $676.82(278.78)$ \\
\hline & Ischaemic stroke & 532 & $2202.96(143.98)$ & 5607.47 (273.97) & $2870.35(281.51)$ & $4572.28(280.27)$ & $682.29(438.78)$ \\
\hline & PTCA/CABG* & 1256 & $1635.76(63.68)$ & $6630.29(208.77)$ & 2121.06 (302.87) & $5823.12(202.55)$ & $598.64(296.25)$ \\
\hline & Heart failure & 1104 & 2737.60 (97.24) & $4818.24(318.44)$ & 5554.17 (1732.62) & 3460.91 (312.14) & 2829.02 (1643.39) \\
\hline & Transient ischaemic attack & 266 & $1999.77(142.44)$ & $2734.81(183.68)$ & 3337.07 (814.53) & $1813.63(187.64)$ & $1691.81(790.76)$ \\
\hline & All patients (hospital) & 5274 & $1588.26(100.82)$ & $4530.08(100.6)$ & 2061.65 (237.53) & $3736.68(85.06)$ & $762.21(237.68)$ \\
\hline & All patients (total) & 5274 & $2380.42(100.97)$ & $5147.79(100.62)$ & $2998.11(242.14)$ & 3967.74 (84.73) & 1017.68 (239.47) \\
\hline \multirow{8}{*}{$\begin{array}{l}\text { First and second } \\
\text { events combined }\end{array}$} & Myocardial infarction & 5237 & 2317.12 (144.72) & $5354.01(113.64)$ & 2472.28 (168.56) & 4277.23 (95.51) & 958.78 (134.53) \\
\hline & Unstable angina & 7148 & 2121.98 (111.68) & 3261.27 (65.83) & 2179.64 (75.16) & $2229.42(50.81)$ & $373.15(74.47)$ \\
\hline & Ischaemic stroke & 4021 & 1994.31 (91.59) & $4533.08(103.14)$ & $2545.1(274.62)$ & 3637.86 (103.05) & 953.48 (261.93) \\
\hline & $\mathrm{PTCA} \mathrm{CABG}^{*}$ & 6338 & $2258.28(69.8)$ & $6802.98(86.73)$ & $1894.5(89.56)$ & 5669.47 (85.13) & $-220.73(83.14)$ \\
\hline & Heart failure & 4700 & 3052.04 (112.99) & 4144.89 (116.03) & $3881.8(344.1)$ & 2635.35 (105.35) & $1128.86(300.08)$ \\
\hline & Transient ischaemic attack & 1923 & $2094.55(195.16)$ & $2519.75(87.9)$ & $2447.92(150.36)$ & $1571.55(68.74)$ & $792.85(146.55)$ \\
\hline & All patients (hospital) & 29367 & $1569.51(43.75)$ & $4133.76(40.95)$ & $1508.48(59.3)$ & 3400.25 (35.58) & 269.32 (53.9) \\
\hline & All patients (total) & 29367 & $2315.55(43.78)$ & $4681.04(41.45)$ & 2351.28 (59.09) & $3576.82(36.18)$ & $438.89(54)$ \\
\hline
\end{tabular}

${ }^{*}$ The proportions of revascularisation events that were CABG were $42 \%$ (2137/5082), 35\% (442/1256) and 41\% (2579/6338) for the first event, second event and all (combined) cohorts, respectively.

CABG, coronary artery bypass graft; CV, cardiovascular; PTCA, percutaneous transluminal coronary angioplasty. 
Figure 2 Incremental costs $(£)$ by Charlson Comorbidity Index score and cohort. Note: Costs in $2014 £$ based on assignment of unit costs to usage as described in the Methods section. Patients stratified by Charlson Comorbidity Index score, which is a function of the number of comorbid conditions and their association with mortality. Higher scores indicate a higher mortality risk.

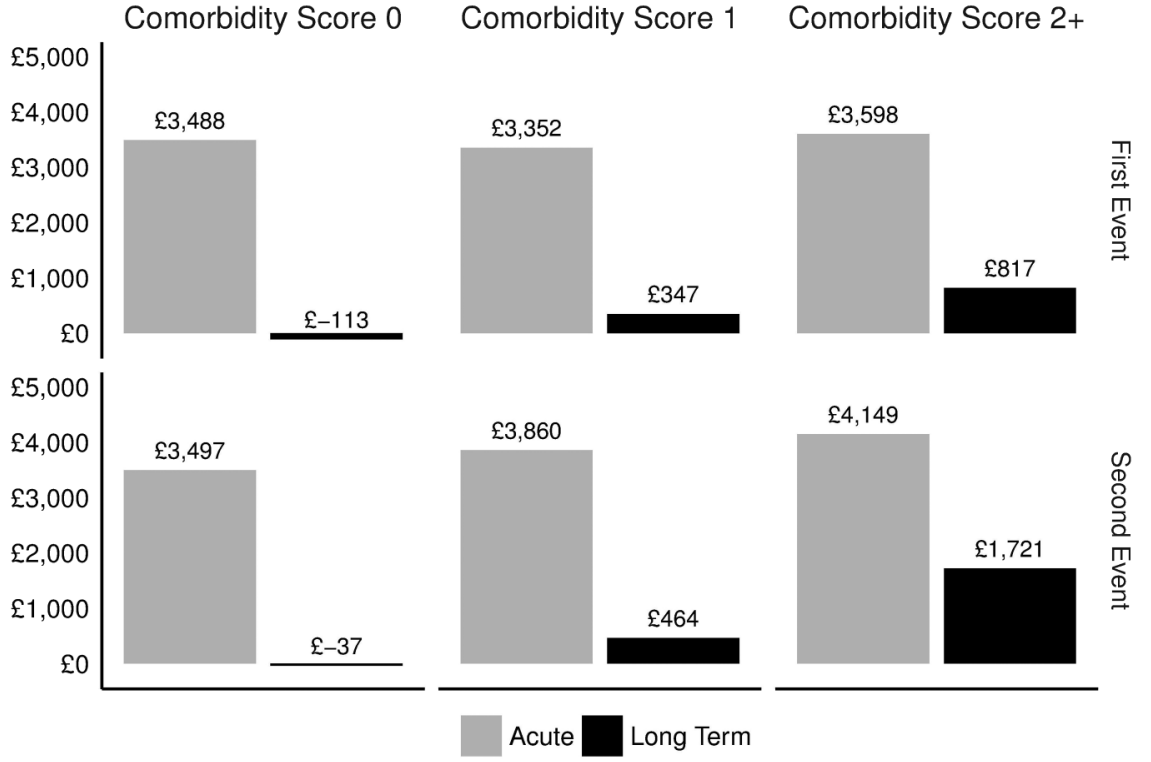

Table 3 Event rates by Charlson Comorbidity Index score and cohort

\begin{tabular}{|c|c|c|c|c|c|c|}
\hline & \multicolumn{2}{|c|}{ Comorbidity score 0} & \multicolumn{2}{|c|}{ Comorbidity score 1} & \multicolumn{2}{|c|}{ Comorbidity score $2+$} \\
\hline & Acute & Long term & Acute & Long term & Acute & Long term \\
\hline \multicolumn{7}{|l|}{ First event } \\
\hline CV hospitalisation LOS (mean days) & 7.6 & NA & 9.6 & NA & 11.4 & NA \\
\hline Office visits (per person year) & 23.2 & 18.1 & 26.6 & 22.9 & 31.8 & 29.7 \\
\hline CV event rate (per 100 person years) & 23.4 & 6.0 & 23.4 & 9.5 & 28.8 & 12.2 \\
\hline Death rate (per 100 person years) & 13.5 & 2.4 & 22.7 & 4.8 & 42.0 & 8.4 \\
\hline \multicolumn{7}{|l|}{ Second event } \\
\hline CV hospitalisation LOS (mean days) & 6.5 & NA & 8.5 & NA & 11.1 & NA \\
\hline Office visits (per person year) & 26.1 & 30.8 & 31.3 & 41.3 & 38.2 & 54.6 \\
\hline CV event rate (per 100 person years) & 25.5 & 11.1 & 38.0 & 12.6 & 39.1 & 19.6 \\
\hline Death rate (per 100 person years) & 8.7 & 1.4 & 24.3 & 3.3 & 48.6 & 9.2 \\
\hline
\end{tabular}

and hospitalisations for other non-CV diseases. However, we have minimised this with a pre-post design over a relatively short period of 3 years, and by calculating incremental costs. In terms of medications, we focused on antithrombotic, antihypertensive and antidiabetic drugs, which are likely to be important. We did not analyse every prescription in the CPRD data due to the variety of drugs, doses and prescriptions; therefore, the drug costs may be underestimated. Drug costs are generally low, so this is not likely to be a large bias. Also, if the initial CV event led to additional resource usage for other reasons (eg, incidentally discovered conditions), these would be included in the incremental costs. Along these lines, older patients may receive different care than younger patients, so the incremental costs might vary by age.

Finally, we used inverse probability of censoring weights to combine data across time periods to reduce the loss of information about costs and the incremental cost of $\mathrm{CV}$ events. However, information from patients who did not survive, or who were censored, is still lost and is estimated by using the data from the remaining patients in the study.
For patients censored due to the end of their data, the missing data are likely to be missing at random and should not introduce a substantial bias. However, for patients who died or were censored due to a subsequent CV hospitalisation, the costs estimated in this study may underestimate the actual cost of care over time if the patients who were censored were higher cost patients. This may at least partly explain the lower cost estimates in the oldest patients (see online supplementary materials figure $\mathrm{S} 2$ ).

Overall, this analysis provides a real-world, evidencebased analysis of the resources used, and the associated costs, in managing CV events in the UK. The burden is higher in higher risk patients. The results demonstrate the substantial economic burden and unmet medical need for patients with CV events in the UK, despite the use of lipid-modifying therapies.

Author affiliations

${ }^{1}$ Outcomes Insights, Inc., Outcomes Research, Westlake Village, California, USA

${ }^{2}$ Amgen (Europe) $\mathrm{GmbH}$, Health Economics, Zug, Switzerland

${ }^{3}$ University of Oxford, Oxford, UK 
${ }^{4}$ Amgen Ltd, Health Economics, Uxbridge, Middlesex, UK

${ }^{5}$ Diabetes Research Centre, University of Leicester, Leicester, UK

${ }^{6}$ St George's, University of London, London, UK

${ }^{7}$ Department of Medicine, School of Public Health, Imperial College London, London, UK

Contributors All the authors contributed to the study design and revisions of the manuscript. MDD, MG and RIG performed the analyses. MDD and MG provided the initial draft. All the authors gave their approval for the final version of the manuscript. All the authors agree to be accountable for all aspects of the manuscript.

Funding This study was funded by Amgen. KK participation was supported by the National Institute for Health Research (NIHR) Collaboration for Leadership in Applied Health Research and Care in East Midlands (CLAHRC EM).

Disclaimer The views expressed are those of the authors and not necessarily those of the NHS, the NIHR or the Department of Health.

Competing interests MDD, MG and RIG work with Outcomes Insights, which was funded to conduct this study. LK and AA are employees of Amgen. KK has acted as a consultant and speaker for Novartis, Novo Nordisk, Sanofi-Aventis, Lilly, Merck Sharp \& Dohme, Janssen, Astra Zeneca and Boehringer Ingelheim. He has received grants in support of investigator and investigator-initiated trials from Novartis, Novo Nordisk, Sanofi-Aventis, Lilly, Pfizer, Boehringer Ingelheim, Merck Sharp \& Dohme, Janssen and Roche, and has served on advisory boards for Lilly, Sanofi-Aventis, Merck Sharp \& Dohme, Novo Nordisk, Boehringer Ingelheim, Janssen and Astra Zeneca. SRKS has provided consulting services to Amgen and received grants from Kowa and Sanofi. KKR has provided consulting services to Amgen, Sanofi, Pfizer, Regeneron, Astra Zeneca, Kowa, Aegerion, Merck Sharp \& Dohme, Lilly and ISIS, and received grants from Sanofi, Regeneron, Amgen, Pfizer and Merck Sharp \& Dohme through his institution.

Provenance and peer review Not commissioned; externally peer reviewed.

Data sharing statement No additional data are available.

Open Access This is an Open Access article distributed in accordance with the Creative Commons Attribution Non Commercial (CC BY-NC 4.0) license, which permits others to distribute, remix, adapt, build upon this work noncommercially, and license their derivative works on different terms, provided the original work is properly cited and the use is non-commercial. See: http:// creativecommons.org/licenses/by-nc/4.0/

\section{REFERENCES}

1. Perk J, De Backer G, Gohlke H, et al, European Association for Cardiovascular Prevention \& Rehabilitation (EACPR); ESC Committee for Practice Guidelines (CPG). European guidelines on cardiovascular disease prevention in clinical practice (version 2012). The Fifth Joint Task Force of the European Society of Cardiology and Other Societies on Cardiovascular Disease Prevention in Clinical Practice (constituted by representatives of nine societies and by invited experts). Eur Heart J 2012;33:1635-701.

2. Baigent C, Blackwell L, Emberson J, et al, Cholesterol Treatment Trialists' (CTT) Collaboration. Efficacy and safety of more intensive lowering of LDL cholesterol: a meta-analysis of data from 170,000 participants in 26 randomised trials. Lancet 2010;376:1670-81.

3. JBS3 Board. Joint British Societies' consensus recommendations for the prevention of cardiovascular disease (JBS3). Heart 2014;100 (Suppl 2):ii1-67.

4. National Institute for Health and Care Excellence. NICE clinical guideline CG181. Lipid modification: cardiovascular risk assessment and the modification of blood lipids for the primary and secondary prevention of cardiovascular disease. Appendices. UK: National Clinical Guideline Center, 2014:589 (accessed 20 Jan 2015).

5. Chapman RH, Liu LZ, Girase PG, et al. Determining initial and follow-up costs of cardiovascular events in a US managed care population. BMC Cardiovasc Disord 2011;11:11.

6. Ohsfeldt RL, Gandhi SK, Fox KM, et al. Medical and cost burden of atherosclerosis among patients treated in routine clinical practice. $J$ Med Econ 2010;13:500-7.

7. Duh MS, Fulcher NM, White LA, et al. Costs associated with cardiovascular events in patients with hypertension in US managed care settings. J Am Soc Hypertension 2009;3:403-15.

8. Eisenstein EL, Shaw LK, Anstrom KJ, et al. Assessing the clinical and economic burden of coronary artery disease: 1986-1998. Med Care 2001;39:824-35.

9. Etemad LR, McCollam PL. Total first-year costs of acute coronary syndrome in a managed care setting. J Manag Care Pharm 2005;11:300-6.

10. Straka RJ, Liu LZ, Girase PS, et al. Incremental cardiovascular costs and resource use associated with diabetes: an assessment of 29,863 patients in the US managed-care setting. Cardiovasc Diabetol 2009;8:53.

11. Herrett E, Gallagher AM, Bhaskaran K, et al. Data Resource Profile: Clinical Practice Research Datalink (CPRD). Int J Epidemiol 2015:44:827-36.

12. Williams $\mathrm{T}$, van Staa $\mathrm{T}$, Puri $\mathrm{S}$, et al. Recent advances in the utility and use of the General Practice Research Database as an example of a UK Primary Care Data resource. Ther Adv Drug Saf 2012;3:89-99.

13. http://www.hscic.gov.uk/qofbrv28 (accessed 20 Jan 2015).

14. https://www.gov.uk/government/uploads/system/uploads/attachment data/file/397469/03a_2013-14_National_Schedule_-_CF-NET_ updated.xIs (accessed 20 Jan 2015).

15. http://www.hscic.gov.uk/casemix/costing (accessed 20 Jan 2015).

16. http://www.pssru.ac.uk/project-pages/unit-costs/2014/ (accessed 20 Jan 2015).

17. http://www.ppa.org.uk/edt/September_2014/mindex.htm (accessed 20 Jan 2015).

18. Charlson ME, Pompei P, Ales KL, et al. A new method of classifying prognostic comorbidity in longitudinal studies: development and validation. J Chronic Dis 1987:40:373-83.

19. Kahn N, Perera R, Harper S, et al. Adaptation and validation of the Charlson Index for Read/OXMIS coded databases. BMC Fam Pract 2010;11:1.

20. Lin DY. Linear regression analysis of censored medical costs Biostatistics 2000;1:35-47.

21. Griffiths RI, Gleeson ML, Danese MD, et al. Inverse probability weighted least squares regression in the analysis of time-censored cost data: an evaluation of the approach using SEER-Medicare. Value Health 2012;15:656-63.

22. Siebert U, Alagoz O, Bayoumi AM, et al. State-transition modeling: a report of the ISPOR-SMDM modeling good research practices task force-3. Med Decis Making 2012;32:690-700.

23. Comprehensive R Archive Network. http://cran.r-project.org (accessed 15 Sep 2015).

24. Nordstrom Beth L, Collins Jenna M, Donaldson R, et al. Treatment patterns and lipid levels among patients with high-risk atherosclerotic CVD in the UK. Br J Cardiol 2015 22:147-54.

25. Herrett E. Validation and validity of diagnoses in the General Practice Research Database: a systematic review. Br J Clin Pharmacol 2009;69:4-14.

26. Clinical Practice Research Datalink. http://www.cprd.com/intro.asp (accessed 15 Sep 2015).

27. Herrett $\mathrm{E}$, Shah $\mathrm{AD}$, Boggon $\mathrm{R}$, et al. Completeness and diagnostic validity of recording acute myocardial infarction events in primary care, hospital care, disease registry, and national mortality records cohort study. BMJ 2013;346:f2350. 